\title{
Transcranial direct current electrical stimulation in combination with aerobic exercise is effective in reducing fatigue and pain in post-COVID-19 systemic autoimmune rheumatic patients
}

Rafael Giovane Missé ( $\nabla$ rafael.gmisse@fm.usp.br)

University of Sao Paulo https://orcid.org/0000-0002-0386-3691

Alexandre Moura dos Santos

University of Sao Paulo https://orcid.org/0000-0002-6998-6723

Isabela Bruna Pires Borges

University of Sao Paulo https://orcid.org/0000-0002-5207-7603

Marlise Sítima Mendes Simões

University of Sao Paulo

Lorenza Rosa Silverio Silva

University of Sao Paulo

Bruna Lindoso Correia

University of Sao Paulo

Ana Wook Sook Kim

University of Sao Paulo

Aline Marques Caetano University of Sao Paulo

Sandra Gofinet Pasoto University of Sao Paulo

Carla Gonçalves Schahin Saad University of Sao Paulo

Diogo Souza Domiciano University of Sao Paulo Clarice Tanaka University of Sao Paulo Júlia Maria D’Andrea Greve University of Sao Paulo Abrahão Fontes Baptista Federal University of ABC Samuel Katsuyuki Shinjo University of Sao Paulo 


\section{Research Article}

Keywords: Autoimmune diseases, COVID-19, fatigue, neuromodulation, physical function, rheumatic diseases, pain

Posted Date: October 25th, 2021

DOI: https://doi.org/10.21203/rs.3.rs-1008362/v1

License: (c) (1) This work is licensed under a Creative Commons Attribution 4.0 International License. Read Full License 
Transcranial direct current electrical stimulation in combination with aerobic exercise is effective in reducing fatigue and pain in post-COVID-19 systemic autoimmune rheumatic patients

\section{Abstract}

Objectives: Currently, no studies have assessed the efficacy of transcranial direct current stimulation (tDCS) to treat fatigue and pain associated with or potentiated by post-COVID-19 syndrome in patients with systemic autoimmune rheumatic diseases (SARDs). Methods: This study included nine patients with different types of SARDs. All patients had previously confirmed RT-PCR test for COVID-19 as well as significant, persistent fatigue and pain that began to worsen after infection. Anodal tDCS was administered in five daily sessions (2mA, 20 minutes) over the $\mathrm{C} 3$ or C4 10/20 EEG positions, with the cathode placed at Fp1 or Fp2. Concomitantly, patients were involved in an aerobic exercise program by treadmill walking. All participants were evaluated using specific questionnaires and strength assessment by handgrip and physical function by timed-up-and go test and sit-to-stand test at baseline (within one week before tDCS protocol), and one week after tDCS protocol. During all procedures, the patients' treatments remained unchanged. Results: The sample was composed of eight women and one man with a mean age of $48.7 \pm 9.6$. After the tDCS protocol, the pain and fatigue significantly improved by a visual analog scale $(P<0.05)$. The physical function also improved $9.5 \pm 2.7 v s .6 .8 \pm 0.8(P=0.001)$ for timed-up-go-test and 10.3 \pm 3.7 vs. $15.1 \pm 4.0(P=0.037)$ for sit-to-stand test. No subject experienced adverse events. One-hundred percent of patients adhered to all protocols. Conclusions: The present study showed tDCS in combination with aerobic exercise was effective in improving the physical function, and reducing fatigue/pain in SARDs patients with post-COVID-19 syndrome.

Clinical Trials: NCT04890483.

Key-words

Autoimmune diseases; COVID-19; fatigue; neuromodulation; physical function; rheumatic diseases; pain. 


\section{Introduction}

The World Health Organization (WHO) declared COVID-19, which is caused by the SARS-CoV-2 infection, a pandemic in March 2020 [1]. The rapid spread of COVID-19 around the world has resulted in at least 230 million infections and deaths as of September 2021 [2]. In addition, about one-third of recovered individuals have several persistent symptoms called long-COVID-19 or post-COVID-19 syndrome [3]. The syndrome is characterized by signs or symptoms that develop during or after SARS-CoV-2 infection and that persist for more than 12 weeks after the COVID-19 recovery period $(3,4)$. Fatigue and musculoskeletal pain have been observed in at least one-third and one-fifth of patients with post-COVID-19 syndrome, respectively [3,4]. As a result, recent studies pointed to the growing dependence of these patients on health systems due to the slow recovery of quality of life and functionality [3].

Systemic autoimmune rheumatic diseases (SARDs) comprise a broad group of diseases characterized by multiorgan and systemic involvement, such as rheumatoid arthritis, systemic lupus erythematosus, Sjögren's syndrome, spondyloarthritis, and systemic autoimmune myopathies. Frequently, patients with SARDs complain of fatigue and chronic pain that negatively affect their quality of life [5-7]. In the context of COVID-19, these individuals could experience aggravated symptoms during and/or after SARS-CoV-2 infection. Indeed, a recent study has shown that patients with chronic pain (e.g., fibromyalgia) experienced worsening of fatigue and pain after COVID-19 [8]. Moreover, these symptoms can persist chronically despite the appropriate regular pharmacological treatments and physical exercise [5-7]. Therefore, it is essential to establish strategies that can reduce chronic pain and fatigue to minimize the impairment of functional capacity of these patients [9-11].

Transcranial direct current electrical stimulation (tDCS) is a noninvasive brain stimulation technique that has shown promising results on pain modulation, fatigue reduction, and improvement in functional capacity in patients with regional complex pain [12,13], refractory myofascial syndrome, chronic fatigue [15], and fibromyalgia [12]. Recently, Pinto et al. [14] showed that tDCS was largely effective in decreasing fatigue in patients with Sjögren's syndrome.

To date, there is a lack of information on tDCS in patients with other SARDs as well as those with postCOVID-19 syndrome. In addition, tDCS in combination with treadmill walking exercise was also effective in relieving refractory myofascial syndrome and chronic fatigue [15].

Because no studies are available, to our knowledge, we aimed to investigate the effectiveness of tDCS in combination with aerobic exercise training on pain and fatigue in SARDs patients with post-COVID-19 symptoms. 


\section{Patients and Methods}

Study design. This is a single-center, prospective open-label one-arm study that included adult SARDs patients with fatigue or pain associated with or potentiated by post-COVID-19 syndrome. The clinical trial was conducted from May to July 2021, and individuals regularly followed in our tertiary outpatient rheumatology clinics were consecutively invited to participate in the study. Currently, our center follows approximately 800 patients with rheumatoid arthritis, 1000 with systemic lupus erythematosus, 220 with systemic autoimmune myopathies, 280 with Sjögren’s syndrome, and 500 with spondyloarthritis.

The study was conducted according to the Declaration of Helsinki and local regulations. It was approved by the local ethical committee (CAAE 41916820.3.0000.0068), and registered in ClinicalTrials (NCT04890483). All patients signed an informed consent form.

Fatigue or pain associated with or potentiated by COVID-19. Post-COVID-19 syndrome was defined as new or worsened fatigue or pain (local or diffuse) sustained for more than 12 weeks after the SARS-CoV-2 infection.

Inclusion criteria. SARDs patients who fulfilled the following classification criteria were recruited: rheumatoid arthritis [17], systemic lupus erythematosus [18], systemic autoimmune myopathies [19], Sjögren's syndrome [20], and spondyloarthritis [21]; COVID-19 confirmed by real-time RT-PCR or serology for IgG and IgM (16) from March 2020 to February 2021; persistent fatigue or pain associated with or potentiated by COVID19 (post-COVID-19 syndrome), defined by new or worsened fatigue, or pain (local or diffuse) sustained for more than 12 weeks after the SARS-CoV-2 infection; relatively stable use of medications in the prior months.

Exclusion criteria. Patients with neoplasms, cardiac pacemaker users, users of cranial metal clips or prostheses, pregnancy, personal history of seizures or epilepsy (related or not to COVID-19), use of drugs that lower seizure threshold, cutaneous lesion (scalp, in the area of application of the electrodes) (Figure 1).

Two experienced rheumatologists and a rheumatology physician with extensive experience with patients with COVID-19 performed the patient eligibility procedure.

Assessments. The following data were collected within one week before the tDCS intervention (Pre-tDCS):

- Demographic: age and gender;

- Anthropometric: body mass index;

- Physical activity level: international physical activity questionnaire (IPAQ), which classifies the level of physical activity and the weekly metabolic equivalent (METs), such as: METs as high physical activity score patients that comply vigorous physical activity $(\geq 5$ days/week and $\geq 30$ minutes per session or vigorous activities during ( $\geq 3$ days/week and $\geq 20$ minutes) and concomitant moderate physical activities ( $\geq 5$ days/week 
and $\geq 30$ minutes per session); moderate activity score - vigorous activities ( $\geq 3$ days/week and $\geq 20$ minutes per session) or moderate physical activities ( $\geq 5$ days/week and $\geq 30$ minutes per session), or the sum of any activities performed $\geq 5$ days/week, and $\geq 150$ minutes/week $[22,23]$;

- Comorbidities: systemic arterial hypertension, dyslipidemia, diabetes mellitus, depression, anxiety, and fibromyalgia. Fibromyalgia was defined according to the American College of Rheumatology classification criteria for fibromyalgia [24]. Depression and anxiety were based on the criteria defined by the American Psychiatric Association [25];

- Pharmacological treatment: current treatment (glucocorticoids and immunosuppressive drugs) and associated medications;

- SARDs activity status was assessed using the composed indices of activity validated for each disease (Clinical Disease Activity Index (CDAI) / Disease activity score (DAS)-28 [27], Sjögren's Syndrome Disease Activity Index (ESSDAI) [28], Bath Ankylosing Spondylitis Activity Index (BASDAI) [29], Manual Muscle Testing-8 (MMT-8) [30], Systemic Lupus Erythematosus Disease Activity Index (SLEDAI) [31];

- Fatigue status and severity were assessed using the VAS, Fatigue Assessment of Chronic Illness Therapy (FACIT-F) [32]; Modified Fatigue Impact Scale (MFIS) [33]; Fatigue Severity Scale (FSS) [34];*

- Pain and fatigue diary: performed during the tDCS and training sessions for aerobic exercise, at baseline and session by session by VAS before each tDCS/aerobic exercise protocol;

- Physical function: Timed Up and Go (TUG) test [35]; Stand-to-Sit test (STS) [36])

- -Muscle strength: handgrip test [37];*

- Quality of life: EQ-5D [38].*

* These data were also collected within one week (Figure 2). During all protocols, the patients' treatments remained unchanged.

Procedures. tDCS. The anode electrode was sourced from a battery-powered direct current generator (Activadose II, USA) and exerted by two electrodes measuring $5 \times 7 \mathrm{~cm}\left(35 \mathrm{~cm}^{2}\right)$ (Ibramed, Brazil) covered by sponge with saline solution and fixed to the head using Velcro straps. Electrodes accepted in accordance with the International System 10/20. Targeting the primary motor cortex, positioned in C3 or C4 10/20 EEG positions, with the cathode placed in the supraorbital region (Fp1 or Fp2, respectively). The active current of tDCS was applied with an electrical current intensity of $2 \mathrm{~mA}$ and a density of $0.057 \mathrm{~mA} / \mathrm{cm}^{2}$ with a duration of 20 minutes, with an up and down ramp of 10 seconds. 
Aerobic exercise. It was characterized by walking on a treadmill for 30 minutes with intensity adjusted through measurement of effort (RPE) during and after 20 minutes of tDCS sessions. In addition, the perception of recovery (PSR) was collected between sessions [39].

Adverse events. They were registered during and after each application through the questionnaire related to the sensations such as burning, tingling, itching, burning (head), headache, nausea, fatigue, emotional lability, difficulty in concentrating and nervousness.

Adherence. Adherence to the protocol was assessed through the registration of controlled gifts by the researchers of this study.

Statistical analysis. Data distribution was alpha $=0.5$. To analyze differences between the clinical characteristics between groups, one-way ANOVA with adjusted factor by group or Friedmann test were used, as posteriori analysis was conducted using a Tukey SD test for multiple comparison. $\mathrm{P}$ value was considered statistically significant when $<0.05$. The individual analysis for the Pre-tDCS and Post-tDCS values were expressed by percentual change by the formula ((Post - Pre) / Pre) x 100. The effect size (ES), a measure of the magnitude of change, was also calculated using Cohen's d for FACIT and MFIS scores and their domains. The software used was SPSS version 25 (Chicago, IL, USA). 


\section{Results}

Of 2800 SARDs patients followed at our clinics, from April 2020 to February 2021, 92 had COVID-19. Seventeen patients showed new worsening or pain and fatigue for more than 12 weeks following SARS-CoV-2 infection. Eight patients refused to participate in the present study (Figure 1). Therefore, nine patients were included in the present study: three with rheumatoid arthritis, two with spondyloarthritis, two with primary Sjögren's syndrome, one with antisynthetase syndrome, and one with systemic lupus erythematosus. These nine patients, whose general features of the participants are shown in Table 1. Mean VAS scores for pain and fatigue were $5.3 \pm 2.7$ and $5.4 \pm 1.8$, respectively. Concerning the quality-of-life parameters, the patients had a current EQ-5D VAS of $5.3 \pm 2.3$ (Figure 2).

At their assessment, we classified two patients (\#1 and \#4) as being in clinical remission, three patients (\#5, $\# 8$, and \#9) with mild disease activity, and three patients (patients \#2, \#3 and \#6) with high underlying disease activity. All patients, regardless of their underlying disease status, reported worsening fatigue or pain after infection with COVID-19.

After tDCS intervention in combination with aerobic exercise training sessions, we observed significant decreases in fatigue and pain VAS scores $(\mathrm{P}<0.05)$ (Figure 2). No changes were observed related to fatigue scores and domains assessed by FACIT and MFIS. Nonetheless, we observed a high ES of 1.00 (CI 95\% $0.80-1.90)$ in the general FACIT scores. Findings were similar for the physical domains in the same questionnaire: ES of 0.80 (CI 95\% -0.16 - 1.70). In contrast, the other FACIT domains the ES for emotional well-being was low: ES of 0.66 (CI 95\% -0.28 - 1.50); the ES for functional well-being was very low, ES of 0.38 (CI 95\% - 0.55 - 1.30), and a very low ES was seen for family well-being, ES: 0.16 (CI 95\% -0.77 - 1.12).

Concerning the general MFIS, the ES was large. -0.81 (CI 95\% $1.10-0.29)$, as well as the physical domains, ES of -0.81 (CI 95\% - $1.77-0.28$ ). The ES was low for cognitive domains, ES of 0.11 (CI 95\% -1.1 - 0.80), and a very low ES was observed for psychosocial domains, ES of -0.19 (CI 95\% -0.1 - 0.8).

During all tDCS interventions, the intensity of the aerobic exercise did not change (mean exercise intensity was $5.5 \pm 0.8$ ), and the patients' perception of recovery through exercise remained unchanged during the protocol. Furthermore, there were no adverse effects of tDCS. In all protocols, $100 \%$ patient adherence. 


\section{Discussion}

To the best of our knowledge, this is the first study to demonstrate the efficacy of tDCS in combination with aerobic exercise training in reducing pain and fatigue after COVID-19 in patients with SARDs.

We conducted a prospective analysis with a well-defined study design that included selected patients with SARDs. To mitigate the risk of bias, patients' pharmacological therapy was unchanged and the patients did not engage in other non-pharmacological interventions outside of the aerobic exercise training program. In addition, we followed up with all patients instant messaging and regularly scheduled face-to-face interviews.

In the analyzed patients who had been classified as in clinical remission the underlying disease (patients \#1 and \#4) remained inactive, with no observed recurrence of disease. Activity rates in the two patients had mild disease activity (patients \#5 and \#8) remained level, with no clinical worsening. A patient (patient \#2) who had been classified as having high disease activity, maintained a high activity rate, with a slight improvement from the initial value. Patients \#3 and \#6, both classified as having high disease activity in the initial assessment, radically changed their indexes, reaching metric values of remission in their final assessment, even though treatment of the underlying diseases did not change during the execution of the study. As the perception of pain is extremely subjective and there were no variations in the laboratory data used, we believe that the worsening of this variable after COVID-19 negatively affected the disease activity indexes. These two cases demonstrate that after treatment with tDCS, improvement in pain was reflected in improvement in disease activity data. Only patient \#9 reported a worsening of clinical joint symptoms after using tDCS; her ESSDAI changed from mild to moderate activity. Patient \#8 could not have her final data evaluated because she was in social isolation due to contact with a family member with a recent diagnosis of COVID-19.

It is important to emphasize that no changes were made in the patients' disease-modifying drug protocols throughout the course of tDCS treatment. Because the application of neurostimulation with tDCS took place for only five days, we chose to not to change the treatments for autoimmune diseases, so that possible positive findings of improvement in relation to pain and/or fatigue would not be affected by other treatment changes. Therefore, we believe that the improvement in the pain and fatigue VAS indexes was correlated with the use of neurostimulation.

tDCS was applied as a non-pharmacological intervention with notable results in the management of several chronic pain syndromes, such as fibromyalgia [40]. Beyond the effects on pain, a significant amount of evidence demonstrates improvements in physical function, mood and health-related quality of life [40,41]. Concerning SARDs, one recent study showed notable improvements in fatigue related to primary Sjögren's syndrome [42], with no adverse effects related to tDCS and the disease status parameters. This result suggests tDCS is a potentially safe and efficient way to improve fatigue in patients with Sjögren's syndrome [42]. However, the authors applied 
tDCS with no other concomitant interventions [42]. Previous studies have shown that tDCS with a concomitant non-pharmacological strategy (e.g., cognitive, motor task or exercise training) facilitated identification of the tDCS target levels that most effectively produce priming effects. Priming effects are the result of increased functional connectivity due to neurotransmitter release, which cases increased cortical excitability in the primary cortex and influences pain processing [43-45]. Moreover, evidence has shown that aerobic exercise training in conjunction with tDCS affects the motor cortex and can result in a major hypoalgesia response to pain related to fibromyalgia [43]. These previous studies support our findings related to improvements in overall pain and fatigue, as measured by VAS. Interestingly, significant improvements were evidenced in physical function. Similar results were reported in patients with stroke [46] and Parkinson's disease [47], suggesting tDCS potentially affects physical function scores [48].

The present study's limitations include its limited sample size and lack of a control group. An observation worthy of attention is that only one patient reported new symptoms of persistent fatigue and pain, whereas all the other patients reported worsening of these symptoms after SARS-CoV-2 infection. Although no studies in the literature that COVID-19 exacerbates pain and fatigue in patients with SARDs, the hypothesis is plausible given these individuals are more likely to have chronic pain and related symptoms. SARS-CoV-2 infection may be related to one of the components of a vicious cycle of pain, fatigue, decreased physical function, and decreased quality of life that has been observed in various SARDs patients, leading to worse overall symptoms. However, no studies have assessed these relationships. Future studies need to investigate the potential relationship between the disease parameters of post-COVID-19 and potential predictive tDCS response in patients with SARDS and post-COVID-19.

In conclusion, tDCS may be an effective strategy to reduce fatigue and pain triggered or potentiated by COVID-19 and ameliorate global functional as well as quality of life. Moreover, the tDCS in combination with aerobic exercise training leads to improvements in physical function. Additional studies with larger sample sizes and randomized design with objective measures of these outcomes are needed to confirm our findings. 


\section{References}

1. WHO. Coronavirus disease (COVID-19) outbreak. In; 2020

2. WHO [Internet]. World alliance for patient safety: WHO draft guidelines for adverse event reporting and learning systems: from information to action 2021. Available from: https://www.who.int/covid19/information

3. Nalbandian A, Sehgal K, Wan EY (2021) Post-acute COVID-19 syndrome. Nat Med 27:601-605

4. Huang C, Huang L, Wang Y, Li X, Ren L, Gu XL (2021) 6-month consequences of COVID-19 in patients discharged from hospital: a cohort study. Lancet 397:220-232

5. Seifert O, Christoph B (2019) Impact of fatigue on rheumatic diseases. Best Pract Res Clin Rheumatol 33:101435

6. Lampa J (2019) Pain without inflammation in rheumatic diseases. Best Pract Res Clin Rheumatol $33: 101439$

7. Fitzcharles MA, Cohen SP, Clauw DJ, Littlejohn G, Usiu C, Häuser W (2021) Nociplastic pain: towards an understanding of prevalent pain conditions. Lancet 397: 2098-2110

8. Cankurtaran D, Tezel N, Ercan B, Yildiz SY, Akyuz EU (2021) The effects of COVID-19 fear and anxiety on symptom severity, sleep quality, and mood in patients with fibromyalgia: a pilot study. Adv Rheumatol $61: 41$

9. Misse RG, Borges IBP, DOS Santos AM, Gupka L, Shinjo SK (2021) Effect of exercise training on fatigue and pain in patients with systemic autoimmune myopathies: A systematic review. Autoimmun Rev 20:102897

10. Baptista AF, Baltar A, Okano AH, Moreira A, Campos ACP, Fernandes AM, et al (2020) Applications of non-invasive neuromodulation for the management of disorders related to COVID-19. Front Neurol $11: 573718$

11. Silva Filho E, Moura S, Santos AC, Brasileira-Santos MS, Albuquerque (2021) Transcranial direct current stimulation as a strategy to manage COVID-19 pain and fatigue. Rev Assoc Med Bras 67:26-28

12. Khedr EM, Omran E, Ismail NM, El-Hammady DH, Goma SH, Kotb H, et al (2017) Effects of transcranial direct current stimulation on pain, mood and serum endorphin level in the treatment of fibromyalgia: A double blinded, randomized clinical trial. Brain Stimul 10:893-901

13. Lagueux É, Bernier M, Bourgault P, Whittingstall K, Mercier C, Léonard G, et al. (2018) The effectiveness of transcranial direct current stimulation as an add-on modality to graded motor imagery for treatment of complex regional pain syndrome: a randomized proof of concept study. Clin J Pain 34:145-154 
14. Pinto A, Piva SR, Vieira A, Gomes SGCN, Rocha AP, Tavares DRB, et al. (2021) Transcranial direct current stimulation for fatigue in patients with Sjogren's syndrome: A randomized, double-blind pilot study. Brain Stimul 14:141-151

15. Misse RG, Dos Santos AM, De Souza JM, Shinjo SK (2020) Transcranial direct current stimulation improves myofascial pain syndrome and chronic fatigue. Reumatismo 72:186-188

16. Corman VM, Landt O, Kaiser M, Molenkamp R, Meijer A, Chu DK, et al. (2020) Detection of 2019 novel coronavirus (2019-nCoV) by real-time RT-PCR. Euro Surveill 25:2000045

17. Aletaha D, Neogi T, Silman AJ, Funovits J, Felson DT, Bingham CO $3^{\text {rd }}$, et al. (2010) 2010 Rheumatoid arthritis classification criteria: An American College of Rheumatology/European League Against Rheumatism collaborative initiative. Arthritis Rheum 62:2569-25681

18. Petri M, Orbai AM, Alarcón GS, Gordn C, Merrill JT, Fortin PR, et al. (2021) Derivation and validation of the systemic lupus international collaborating clinics classification criteria for systemic lupus erythematosus. Arthritis Rheum 64:2677-2686

19. Lundberg IE, Tjärnlund A, Bottai M, Werth VP, Pilkington C, De Visser M, et al. (2017) 2017 European League Against Rheumatism / American College of Rheumatology classification criteria for adult and juvenile idiopathic inflammatory myopathies and their major subgroups. Arthritis Rheumatol.69:22712282

20. Vitali C, Bombardieri S, Jonsson R, Moutsoupoulos HM, Alexander EL, Carsons SE, et al. (2002) Classification criteria for Sjögren's syndrome: A revised version of the European criteria proposed by the American-European Consensus Group. Ann Rheum Dis 61:554-558

21. Rudwaleit M, Van Der Heijde D, Landewé R, Listing J, Akkoc N, Brandt J, et al. (2009) The development of Assessment of SpondyloArthritis international Society classification criteria for axial spondyloarthritis (part II): Validation and final selection. Ann Rheum Dis 68:777-783

22. Craig CL, Marshall AL, Sjostrom M, Bauman AE, Booth ML, Ainsworth BE, et al. (2020) International physical activity questionnaire: 12-country reliability and validity. Med Sci Sports Exerc 2003;35:13811395

23. Singh R, Pattisapu A, Emery MS (2020) US Physical Activity Guidelines: Current state, impact and future directions. Trends Cardiovasc Med 30:407-412

24. Wolfe F, Clauw DJ, Fitzcharles MA, Goldenberg DL, Katz RS, Mease P, et al. (2010) The American College of Rheumatology preliminary diagnostic criteria for fibromyalgia and measurement of symptom severity. Arthritis Care Res (Hoboken) 62:600-610 
25. American Psychiatric Association. Diagnostic and statistical manual of mental disorders, 5th edition (DSM5). Arlington, VA, USA: American Psychiatric Publishing, 2013

26. Bruce B, Fries J (2003) The Stanford health assessment questionnaire: dimensions and practical applications. Health Qual Life Outcomes 1:20

27. Prevoo MLL, Van’t Hof MA, Kuper HH, Van Leeuwen MA, Van De Putter LB, Van Riel PL (1995) Modified disease activity scores that include twenty-eight-joint counts: development and validation in a prospective longitudinal study of patients with rheumatoid arthritis. Arthritis Rheum 38:44-48

28. Serrano EV, Valim V, Miyamoto ST, Giovelli RA, Paganotti MA, Cadê NV (2013) Transcultural adaptation of the "EULAR Sjögren's Syndrome Disease Activity Index (ESSDAI)" into Brazilian Portuguese. Rev Bras Reumatol 53:483-493

29. Calin A, Garrett S, Whitelock H, Kennedy LG, O’Hea J, Mallorie P, et al. (1994) Av new approach to defining functional ability in ankylosing spondylitis: The development of the Bath Ankylosing Spondylitis Functional Index. J Rheumatol 21:2281-2285

30. Rider LG, Koziol D, Giannini EH, Jain MS, Smith MR, Whtiney-Mahoney K, et al. (2010) Validation of manual muscle testing and a subset of eight muscles for adult and juvenile idiopathic inflammatory myopathies. Arthritis Care Res 62:465-472

31. Gladman DD, Ibanez D, Urowitz MB (2002) Systemic lupus erythematosus disease activity index 2000. J Rheumatol;29:288-291

32. Brucker P, Yost K, Cashy J, Webster K, Cella (2005) General population and cancer patient norms for the Functional Assessment of Cancer Therapy - General (FACT-G). Eva Health Prof 28:192-211

33. Larson RD (2013) Psychometric properties of the modified fatigue impact scale. Int J MS Care 15:15-20

34. Nadarajah M, Mazlan M, Abdul-Latif L, Goh HT (2017) Test-retest reliability, internal consistency and concurrent validity of Fatigue Severity Scale in measuring post-stroke fatigue. Eur J Phys Rehabil Med 53:703-709

35. Podsiadlo D, Richardson S (1991) The timed "Up \& Go": a test of basic functional mobility for frail elderly persons. J Am Geriatr Soc 39:142-148

36. Newcomer KL, Krug HE, Mahowald ML (1993) Validity and reliability of the timed-stands test for patients with rheumatoid arthritis and other chronic diseases. J Rheumatol 20:21-27

37. Innes EV (1999) Handgrip strength testing: a review of the literature. Australian Occup Ther J 46:120-140

38. Greiner W, Weijnen T, Nieuwenhuizen M, Oppe S, Badia X, Busschbach J, et al. (2003) A single European currency for EQ-5D health states. Results from a six-country study. Eur J Health Econom 4:222-2231 
39. Borg GA (1982) Psychophysical bases of perceived exertion. Med Sci Sports Exerc 14:377-381

40. Knotkova H, Hamani C, Sivanesan E, Le Beuffe MFE, Moon JY, et al. (2021) Neuromodulation for chronic pain. Lancet 397:2111-124

41. Baptista AF, Fernandes A, Sá KN, Okano AH, Brunoni AR, Lara-Solares A, et al. (2019) Latin American and Caribbean consensus on noninvasive central nervous system neuromodulation for chronic pain management (LAC2-NIN-CP). Pain Rep 4:e692

42. Pinto A, Piva SR, Vieira A, Gomes SGCN, Rocha AP, Tavares DRB, et al. (2021) Transcranial direct current stimulation for fatigue in patients with Sjögren's syndrome: A randomized, double-blind pilot study. Brain Stimul 14:141-151

43. Mendonca ME, Simis M, Grecco LC, Battistella LR, Baptista AF, et al. (2016) Transcranial direct current stimulation combined with aerobic exercise to optimize analgesic responses in fibromyalgia: a randomized placebo-controlled clinical trial. Front Human Neurosci 10:68

44. Franco KFM, Lenoir D, Franco YRS, Reis FJJ, Cabral CMN, Meeus M (2021) Prescription of exercises for the treatment of chronic pain along the continuum of nociplastic pain: A systematic review with metaanalysis. Eur J Pain 25:51-70

45. Rice D, Nijs J, Kosek E, Wideman T, Hasenbring MI, Koltyn K, et al. (2019) Exercise-induced hypoalgesia in pain-free and chronic pain populations: state of the art and future directions. J Pain 20:1249-1266

46. Elsner B, Kwakkel G, Kugler J, Mehrholz J (2017) Transcranial direct current stimulation (tDCS) for improving capacity in activities and arm function after stroke: a network meta-analysis of randomised controlled trials. J Neuroeng Rehabil 14:95

47. Chen KS, Chen R (2019) Invasive and noninvasive brain stimulation in Parkinson's disease: clinical effects and future perspectives. Clin Pharmacol Ther 106:763-775

48. Dagan M, Herman T, Harrison R, Zhou J, Giladi N, Ruffini G, et al. (2018) Multitarget transcranial direct current stimulation for freezing of gait in Parkinson's disease. Movement disorders. J Mov Dis Soc 2018;33:64264-6

49. Machado D, Unal G, Andrade SM, Moreira A, Altimari LR, Brunoni AR, et al. (2019) Effect of transcranial direct current stimulation on exercise performance: A systematic review and meta-analysis. Brain Stimul $12: 593-605$

50. Fregni F, Gimenes R, Valle AC, Ferreira MJL, Rocha RR, Natalle L, et al. (2016) A randomized, shamcontrolled, proof of principle study of transcranial direct current stimulation for the treatment of pain in fibromyalgia. Arthritis Rheum 54:3988-3998 


\section{Figures}

Figure 1. The study flow-chart

ASSD: antisynthetase syndrome; RA: rheumatoid arthritis; SARDs: systemic autoimmune rheumatic diseases;

SLE: systemic lupus erythematosus: SpA: spondyloarthritis; SSj: Sjögren’s syndrome.

Figure 2. Data related to the general pain, fatigue and quality of life visual analogue scales

EQ-5D: Health-related quality of life; VAS: Visual Analogue Scale.

Figure 3. Data related to the mean fatigue and quality of life scores

EQ-5D: EuroQual. FACIT: Fatigue Assessment of Chronic Illness Therapy. 
Figures

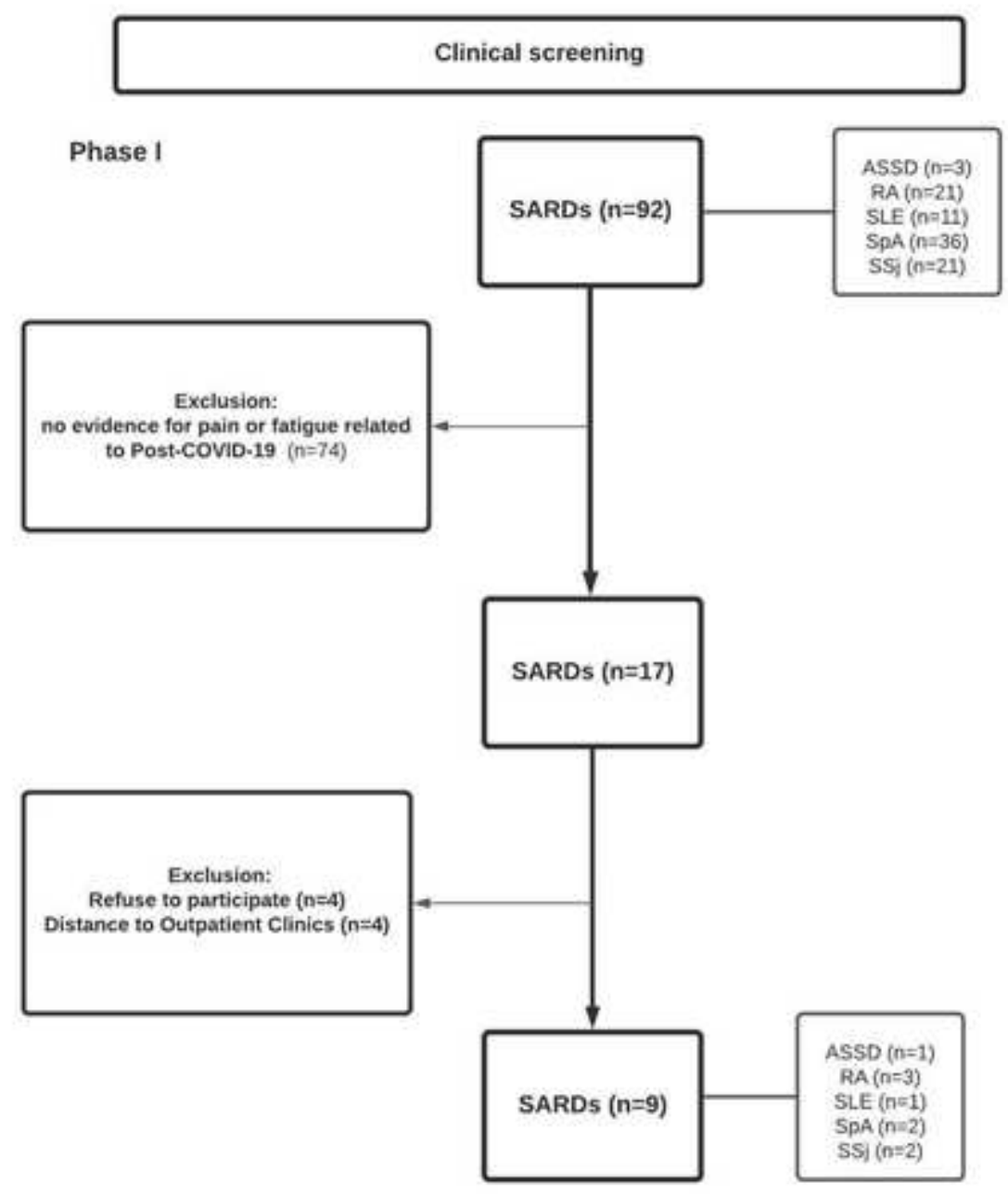

Experimental design

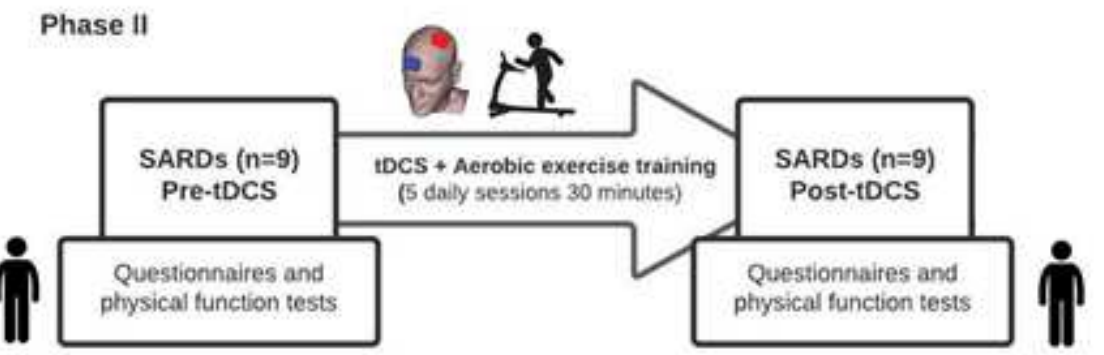

\section{Figure 1}

The study flow-chart ASSD: antisynthetase syndrome; RA: rheumatoid arthritis; SARDs: systemic autoimmune rheumatic diseases; SLE: systemic lupus erythematosus: SpA: spondyloarthritis; SSj: Sjögren's syndrome. 

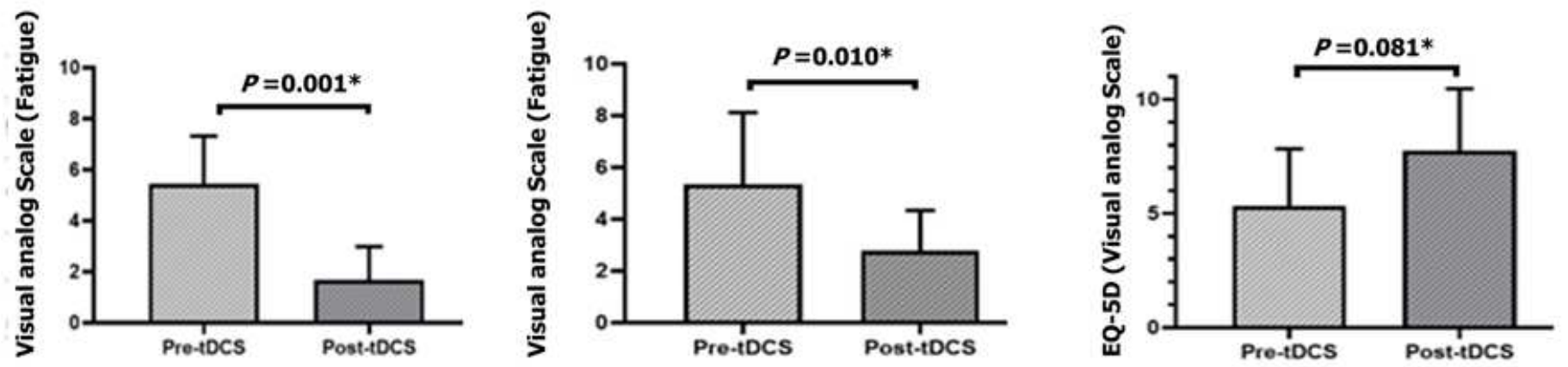

Figure 2

Data related to the general pain, fatigue and quality of life visual analogue scales EQ-5D: Health-related quality of life; VAS: Visual Analogue Scale.
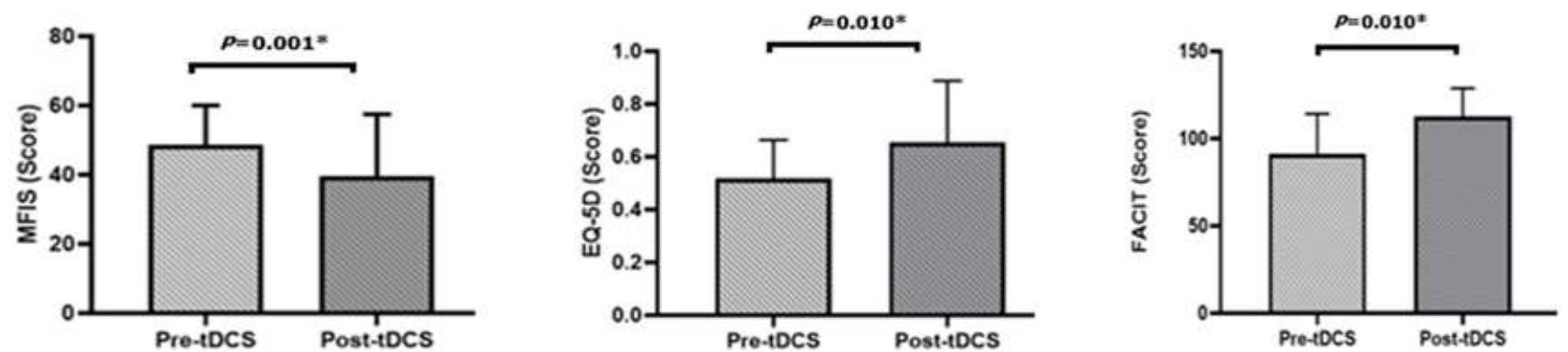

Figure 3

Data related to the mean fatigue and quality of life scores EQ-5D: EuroQual. FACIT: Fatigue Assessment of Chronic Illness Therapy.

\section{Supplementary Files}

This is a list of supplementary files associated with this preprint. Click to download.

- Tables.pdf 